\title{
Projective absoluteness for Sacks forcing
}

\author{
Daisuke Ikegami
}

Received: 12 February 2008 / Revised: 2 July 2009 / Published online: 12 September 2009

C The Author(s) 2009. This article is published with open access at Springerlink.com

\begin{abstract}
We show that $\Sigma_{3}^{1}$-absoluteness for Sacks forcing is equivalent to the nonexistence of a $\Delta_{2}^{1}$ Bernstein set. We also show that Sacks forcing is the weakest forcing notion among all of the preorders that add a new real with respect to $\Sigma_{3}^{1}$ forcing absoluteness.
\end{abstract}

Keywords Forcing absoluteness $\cdot$ Sacks forcing $\cdot$ Bernstein sets

Mathematics Subject Classification (2000) $\quad 03 \mathrm{E} 15 \cdot 28 \mathrm{~A} 05 \cdot 54 \mathrm{H} 05$

\section{Introduction}

Absoluteness is one of the central notions in set theory, which is the unchangingness of the truth-values of statements between two models of set theory. Forcing absoluteness is the absoluteness between ground models and their generic extensions, which plays an important role in many areas in set theory. In this paper, we focus on forcing absoluteness of projective statements (or statements in second-order arithmetic). (For the precise definition of this forcing absoluteness, see Definition 2.14.)

Forcing absoluteness has close connections with regularity properties in descriptive set theory. For example, $\Sigma_{3}^{1}$ statements are absolute between $V$ and its generic extensions by Cohen forcing iff every $\Delta_{2}^{1}$ set of reals has the Baire property iff for any

D. Ikegami $(\varangle)$

The Institute for Logic, Language and Computation, The Universiteit van Amsterdam,

Science Park 904, 1098 XH, Amsterdam, The Netherlands

e-mail:d.ikegami@uva.nl 
real $r$ there is a Cohen real over $\mathrm{L}[r]$. The same kind of equivalence holds for random forcing and Lebesgue measurability. ${ }^{1}$

There is also a relation between forcing absoluteness for other forcings and these regularity properties for $\Sigma_{2}^{1}$ sets of reals, e.g., $\Sigma_{3}^{1}$ statements are absolute between $V$ and its generic extensions by Hechler forcing iff every $\Sigma_{2}^{1}$ set of reals has the Baire property iff for any real $r$, the set of all Cohen reals over $\mathrm{L}[r]$ is comeager. There is also an analogue for amoeba forcing and Lebesgue measurability. ${ }^{2}$

Sometimes, the regularity property for all $\Delta_{2}^{1}$ sets of reals is equivalent to that for all $\Sigma_{2}^{1}$ sets of reals. The Ramsey property is a typical example and it is connected to forcing absoluteness for Mathias forcing: $\Sigma_{3}^{1}$ statements are absolute between $V$ and its generic extensions by Mathias forcing iff every $\Sigma_{2}^{1}$ (or $\boldsymbol{\Delta}_{2}^{1}$ ) set of reals has the Ramsey property iff for any real $r$, there is a Ramsey real over $\mathrm{L}[r]$ (or the set of all reals Ramsey over $\mathrm{L}[r]$ is co-Ramsey). ${ }^{3}$

In this paper, we show that Sacks forcing is this kind of forcing. As a corollary, we see that Sacks forcing is the weakest forcing notion among all of the preorders that add a new real with respect to $\Sigma_{3}^{1}$ forcing absoluteness. More precisely,

\section{Theorem 3.1 Let $\mathbb{S}$ be Sacks forcing.}

1. The following are equivalent:

(a) $\Sigma_{3}^{1}$-S-absoluteness holds.

(b) There is no $\Delta_{2}^{1}$ Bernstein set.

(c) There is no $\boldsymbol{\Sigma}_{2}^{1}$ Bernstein set.

(d) For any real $r$, there is a real $x$ such that $x$ is not in $\mathrm{L}[r]$.

2. Suppose that $\mathbb{P}$ is a preorder which adds a new real (i.e. there is a $\mathbb{P}$-generic filter $G$ over $V$ such that there is a real in $V[G]$ but not in $V)$. Then $\Sigma_{3}^{1}-\mathbb{P}$-absoluteness implies $\Sigma_{3}^{1}$-S-absoluteness.

Note that the equivalence of (b), (c), and (d) of 1 in Theorem 3.1 was already proved by Brendle and Löwe [5] (Theorem 7.1, p. 1321).

Bernstein sets are typical counter examples for every regularity property. Hence, we could say that the property not being a Bernstein set is the weakest regularity property (for the definition of Bernstein sets, see Definition 2.3). There is a corresponding regularity property to not being a Bernstein set so-called Sacks measurability (for the definition, see Definition 2.5). In the proof of Theorem 3.4 (we will state later), we use Sacks measurability to prove forcing absoluteness rather than the non-existence of Bernstein sets.

\footnotetext{
1 The direction from forcing absoluteness to the regularity properties was proved by Bagaria [1] and the converse was proved by Woodin [20]. The equivalence between the regularity properties and the transcendence properties for L was proved by Judah and Shelah [10]. For the entire proofs, see Theorem 9.2.12 (p. 456) and Theorem 9.2.1 (p. 452) in Bartoszyński and Judah [2].

2 The equivalence between forcing absoluteness and the regularity properties was proved by Judah [9] and the equivalence between the regularity properties and the transcendence properties for $\mathrm{L}$ was proved by Solovay [19]. For the entire proofs, see Theorem 9.3 .8 (p. 460) and Theorem 9.3.1 (p. 457) in Bartoszyński and Judah [2].

3 The equivalence between forcing absoluteness and the regularity property was proved by Halbeisen and Judah [7] (Theorem 4.1, p. 187). The other equivalences were proved by Judah and Shelah [10] (2.7 Theorem and 2.8 Theorem, p. 219).
} 
It is a natural question whether these equivalences hold for higher level forcing absoluteness and the regularity properties for more complex projective classes. For the direction from forcing absoluteness to the regularity properties, several results have been established, e.g., if $\mathbb{D}$ is Hechler forcing and $\Sigma_{4}^{1}-\mathbb{D}$-absoluteness holds, then every $\Sigma_{3}^{1}$ set of reals has the Baire property. Furthermore, for any $n \geq 4$, if $\Sigma_{n+1}^{1}$-D-absoluteness and $\Sigma_{n+1}^{1}-\mathbb{D} * \mathbb{D}$-correctness hold, then every $\Sigma_{n}^{1}$ set of reals has the Baire property. Here, forcing correctness is a slightly stronger condition than forcing absoluteness (for the definition, see Definition 2.14). There is also an analogue for amoeba forcing and Lebesgue measurability and there are some partial results for Mathias forcing and the Ramsey property. ${ }^{4}$ (This generalization does not work for Cohen forcing and the Baire property and for random forcing and Lebesgue measurability. ${ }^{5}$ )

However, it is still open whether these regularity properties follow from only forcing absoluteness for arbitrary projective classes. We will show that this is the case for Sacks forcing:

Theorem 3.3 Let $n$ be a natural number with $n \geq 1$. If $\Sigma_{n+1}^{1}$-S-absoluteness holds, then there is no $\Delta_{n}^{1}$ Bernstein set.

We do not know if we can replace $\boldsymbol{\Delta}_{n}^{1}$ by $\boldsymbol{\Sigma}_{n}^{1}$ above.

For the reverse direction (from the regularity properties to forcing absoluteness), there is a little known as negative results with only assuming ZFC. For example, the Baire property for every projective set does not imply $\Sigma_{4}^{1}$ forcing absoluteness for Hechler forcing because the consistency strength of the former statement is just ZFC (7.17 Conclusion (p. 43) in Shelah [17]) but that of the latter is inaccessible (Theorem 9.5.6 (p. 477) in Bartoszyński and Judah [2]). For the same reason, the Ramsey property for every $\boldsymbol{\Delta}_{3}^{1}$ set of reals does not imply $\boldsymbol{\Sigma}_{4}^{1}$ forcing absoluteness for Mathias forcing (Theorem 5.2 (p. 188) in Halbeisen and Judah [7]).

But, if we assume the uniformization property for suitable projective classes, we can get positive results: e.g., if $n \geq 1, \Pi_{2 n-1}^{1}$ has the uniformization property and every $\Delta_{2 n}^{1}$ set of reals has the Baire property, then $\Sigma_{2 n+1}^{1}$ forcing absoluteness for Cohen forcing holds. ${ }^{6}$ (For the definition of the uniformization property, see Definition 2.20.) Here we only assume the uniformization property for odd level projective classes because $\Pi_{2}^{1}$ does not have the uniformization property and these assumptions are true under suitable large cardinals assumptions or projective determinacy. (For the details, see Remark 2.22, Theorem 2.23, and Theorem 2.24.)

We will prove that this is also true for Sacks forcing:

\footnotetext{
${ }^{4}$ Lebesgue measurability for all $\Sigma_{3}^{1}$ sets of reals from $\Sigma_{4}^{1}$ absoluteness for amoeba forcing was proved by Brendle [3]. For the proofs for Lebesgue measurability and the Baire property, see Theorem 9.5.5, Theorem 9.5.6 (p. 476-477) and Theorem 9.6.3 (p. 479) in Bartoszyński and Judah [2]. For the Ramsey property, see Theorem 5.3 (p. 189), Corollary 6.1 (p. 191), and Corollary 6.5 (p.192) in Halbeisen and Judah [7].

5 More precisely, it is consistent with ZFC that every projective statement is absolute between $V$ and its generic extensions by Cohen forcing but there is a $\Sigma_{2}^{1}$ set of reals without the Baire property. The same holds for random forcing and Lebesgue measurability.

6 The same holds for random forcing and Lebesgue measurability. For the proofs, see Lemma 2 (p. 367) in Woodin [20].
} 
Theorem 3.4 Let $n$ be a natural number with $n \geq 1$. Assume that $\boldsymbol{\Pi}_{2 n-1}^{1}$ has the uniformization property. If there is no $\boldsymbol{\Delta}_{2 n}^{1}$ Bernstein set, then $\boldsymbol{\Sigma}_{2 n+1}^{1}$-S-absoluteness holds.

This paper consists of three sections. In the second section we will look at the basic concepts and the facts for our results. In the last section we will prove our results.

\section{Basic concepts and facts}

From now on, we will work in ZFC. We assume that readers are familiar with the elementary theories of forcing and descriptive set theory. (For basic definitions we will not mention, see Jech [8] and Moschovakis [15].) Also when we call something a real, it is an element of Cantor space ${ }^{\omega} 2$. (Usually, we mean an element of Baire space ${ }^{\omega} \omega$ by a real. But for simplicity, we will work on Cantor space and there are no essential differences in the following arguments.)

Notation 2.1 For $s$ in ${ }^{<} 2$, put

$$
N_{s}=\left\{x \in{ }^{\omega} 2 \mid s \subseteq x\right\}
$$

$\left\{N_{s} \mid s \in{ }^{<\omega} 2\right\}$ forms a basis for the topology of Cantor space.

Definition 2.2 Let $X$ be a set and $T$ be a non-empty tree on $X$.

1. For nodes $s, t$ of $T, s, t$ are incompatible in $T$ if there is no node $u$ of $T$ such that $s, t$ are subsequences of $u$.

2. The tree $T$ is perfect if for any node $t$ of $T$, there are two nodes $u, v$ of $T$ such that $t$ is a subsequence of $u, v$ and $u, v$ are incompatible in $T$.

3. Define $[T]$ as follows:

$$
[T] \stackrel{\text { def }}{=}\left\{x \in{ }^{\omega} X \mid(\forall n \in \omega) x\lceil n \in T .\} .\right.
$$

4. When there are at least two nodes in $T$ which are incompatible, let stem $(T)$ denote the maximal node $t_{0}$ of $T$ such that for any node $t$ of $T$, either $t_{0} \subseteq t$ or $t \subseteq t_{0}$ holds.

\section{Definition 2.3 (Bernstein sets)}

1. A set of reals $P$ is perfect if there is a perfect tree $S$ on 2 such that $P=[S]$.

2. A set of reals $P$ is a Bernstein set if neither $P$ nor ${ }^{\omega} 2 \backslash P$ contains a perfect subset of Cantor space.

Remark 2.4 If $P$ is a Bernstein set, then $P$ does not satisfy the Baire property and the Lebesgue measurability.

\section{Definition 2.5 (Sacks measurability)}

1. A set of reals $P$ is Sacks null if for any perfect tree $S$, there is a perfect tree $S^{\prime}$ such that $S^{\prime} \subseteq S$ and $\left[S^{\prime}\right] \cap P=\emptyset$. 
2. A set of reals $P$ is of Sacks measure one if for any perfect tree $S$, there is a perfect tree $S^{\prime}$ such that $S^{\prime} \subseteq S$ and $\left[S^{\prime}\right] \subseteq P$.

3. A set of reals $P$ is Sacks measurable if for any perfect tree $S$, there is a perfect tree $S^{\prime}$ such that $S^{\prime} \subseteq S$ and either $\left[S^{\prime}\right] \cap P=\emptyset$ or $\left[S^{\prime}\right] \subseteq P$ holds.

As we mentioned in Sect. 1, Sacks measurability coincides with the property of not being a Bernstein set in the following sense:

Remark 2.6 Let $n$ be a natural number with $n \geq 1$ and $\Gamma$ denote one of the following pointclasses, $\boldsymbol{\Sigma}_{n}^{1}, \boldsymbol{\Pi}_{n}^{1}$, or $\boldsymbol{\Delta}_{n}^{1}$. Then the following are equivalent:

1. Every set of reals in $\boldsymbol{\Gamma}$ is Sacks measurable.

2. No sets of reals in $\boldsymbol{\Gamma}$ are Bernstein sets.

Proof See Lemma 2.1 (p. 1310) in Brendle and Löwe [5].

Note that typical regularity properties can be expressed in the analogous way to the definition of Sacks measurability as follows:

Remark 2.7 1. A set of reals $P$ has the Baire property iff for any $s$ in ${ }^{<\omega} 2$, there exists an $s^{\prime}$ in ${ }^{<\omega} 2$ such that $s^{\prime} \supseteq s$ and either $N_{s^{\prime}} \cap P$ or $N_{s^{\prime}} \backslash P$ is meager.

2. A set of reals $P$ is Lebesgue measurable iff for any Borel subset $B$ of ${ }^{\omega} 2$ with a positive Lebesgue measure, there exists a Borel subset $B^{\prime}$ of ${ }^{\omega} 2$ with a positive Lebesgue measure such that $B^{\prime} \subseteq B$ and either $B^{\prime} \cap P$ or $B^{\prime} \backslash P$ is null.

We will refer to the following fact, which is a part of Theorem 3.1:

Theorem 2.8 [5, Theorem 7.1, p. 1321] The following are equivalent:

1. There is no $\Delta_{2}^{1}$ Bernstein set.

2. There is no $\Sigma_{2}^{1}$ Bernstein set.

3. For any real $r$, there is a real $x$ such that $x$ is not in $\mathrm{L}[r]$.

Let us review the definition and the basic properties of Sacks forcing:

Definition 2.9 (Sacks forcing) Sacks forcing $\mathbb{S}$ is defined in the following way:

$$
\mathbb{S} \stackrel{\text { def }}{=}\{S \mid S \text { is a perfect tree on } 2\} .
$$

For $S_{1}, S_{2}$ in $\mathbb{S}, S_{1} \leq S_{2}$ if $S_{1} \subseteq S_{2}$.

Remark 2.10 Suppose that $G$ is an $\mathbb{S}$-generic filter over $V$. Put

$$
s=\bigcup\{\operatorname{stem}(S) \mid S \in G\} .
$$

Then, by the genericity of $G, s$ is a real. Such a real is called a Sacks real over $V$.

On the other hand, $G$ is reconstructed from $s$ and $V$ because

$$
G=\{S \in \mathbb{S} \cap V \mid s \in[S]\} .
$$

Therefore, there is a canonical correspondence between Sacks reals over $V$ and $\mathbb{S}$-generic filters over $V$. From now on, we identify Sacks reals over $V$ with $\mathbb{S}$-generic filters over $V$ in the above way. 
The following property is known as the minimality of Sacks forcing:

Theorem 2.11 (Sacks [16]) Suppose that s is a Sacks real over $V$. Then, in $V[s]$, for any set $X$ such that $X$ is not in $V$ and $X \subseteq V, V[X]=V[s]$.

Proof The proof can be found in Lemma 28 (p. 18) in Geschke and Quickert [6].

We will need the following further properties of Sacks forcing:

Theorem 2.12 (Sacks [16]) Suppose that s is a Sacks real over V. Then, in $V[s]$, every real $s^{\prime}$ which is not in $V$ is also a Sacks real over $V$.

Proof The proof can be found in Lemma 27 and the discussion after it (p. 16-17) in Geschke and Quickert [6].

Theorem 2.13 (Brendle [4] (Theorem 4, p. 110)) Suppose that s is a Sacks real over $V$. Then, in $V[s]$, the set

$$
\left\{s^{\prime} \mid s^{\prime} \text { is a Sacks real over } V .\right\}
$$

is of Sacks measure one.

Now we come to the forcing absoluteness which is the main subject of this paper:

Definition 2.14 (Forcing absoluteness) Let $n$ be a natural number with $n \geq 1, \mathbb{P}$ be a preorder, and $\boldsymbol{\Gamma}$ be $\Sigma_{n}^{1}$ or $\Pi_{n}^{1}$.

1. By $\boldsymbol{\Gamma}$ - $\mathbb{P}$-absoluteness, we mean the following statement:

If $G$ is a $\mathbb{P}$-generic filter over $V$, then for any $\boldsymbol{\Gamma}$-formula $\phi$ and any real $x$ in $V$,

$$
V \vDash \phi(x) \text { iff } V[G] \vDash \phi(x) .
$$

2. By $\boldsymbol{\Gamma}$-P-correctness, we mean the following statement:

If $G$ is a $\mathbb{P}$-generic filter over $V$, then for any $\Gamma$-formula $\phi$ and any real $x$ in $V[G]$,

$$
V[x] \vDash \phi(x) \text { iff } V[G] \vDash \phi(x) .
$$

Remark 2.15 Let $n$ be a natural number with $n \geq 1$ and $\mathbb{P}$ be a preorder.

1. $\Sigma_{n}^{1}-\mathbb{P}$-absoluteness is equivalent to $\Pi_{n}^{1}-\mathbb{P}$-absoluteness. The same holds for forcing correctness.

2. $\quad \Sigma_{n}^{1}-\mathbb{P}$-correctness implies $\Sigma_{n}^{1}-\mathbb{P}$-absoluteness.

The following remark shows us why we need only forcing absoluteness for Sacks forcing to prove the non-existence of Bernstein sets:

Remark 2.16 Let $n$ be a natural number with $n \geq 1$. By the minimality of Sacks forcing, $\Sigma_{n}^{1}$-S-correctness is equivalent to $\Sigma_{n}^{1}-\mathbb{S}$-absoluteness.

The following result is a basic tool in descriptive set theory, called "Shoenfield absoluteness": 
Theorem 2.17 (Shoenfield [18]) Suppose $M$ is a transitive model of $Z F+D C$ containing $\omega_{1}^{V}$. Then every $\Sigma_{2}^{1}$-formula is absolute between $M$ and $V$.

Proof The proof can be found in Theorem 25.20 (p. 490) in Jech [8].

Remark 2.18 By Theorem 2.17, we have $\Sigma_{2}^{1}$-P -correctness for any preorder $\mathbb{P}$.

In the proof of Shoenfield absoluteness, he constructed an absolute tree called "Shoenfield tree" for each $\Sigma_{2}^{1}$ statement, which itself is important in descriptive set theory:

Theorem 2.19 (Shoenfield [18]) For any real $a$ and $a \Sigma_{2}^{1}(a)$ set $P$, there is a tree $T$ on $2 \times \omega_{1}$ in $L[a]$ such that $P=p[T]$, where $p[T]$ is the image of $[T]$ via the projection $\pi:{ }^{\omega} 2 \times \omega_{1} \rightarrow{ }^{\omega} 2$.

Proof The proof can be found in Theorem 25.20 (p. 490) in Jech [8].

Now we introduce the uniformization property for proving Theorem 3.4:

Definition 2.20 (Uniformization) Let $\boldsymbol{\Gamma}$ be a subset of $\mathcal{P}\left({ }^{\omega} 2 \times{ }^{\omega} 2\right)$. Then $\boldsymbol{\Gamma}$ has the uniformization property if for any relation $P$ in $\boldsymbol{\Gamma}$, there is a function $f$ from ${ }^{\omega} 2$ into itself in $\Gamma$ (as a graph) such that $f \subseteq P$ (as a graph) and the domain of $f$ is the same as that of $P$.

This definition allows us to replace relations by functions while keeping the complexity of sets, which is often useful in descriptive set theory.

The following is a classical result on the uniformization property:

Theorem 2.21 (Kondo [11]) The pointclasses $\boldsymbol{\Pi}_{1}^{1}$ and $\boldsymbol{\Sigma}_{2}^{1}$ have the uniformization property.

Proof The proof can be found in 4E.4 (p. 235) in Moschovakis [15].

Remark 2.22 Let $\boldsymbol{\Gamma}$ be a projective pointclass. If $\boldsymbol{\Gamma}$ has the uniformization property, then the dual class of $\boldsymbol{\Gamma}$ (i.e. the set of complements of sets in $\boldsymbol{\Gamma}$ ) does not have the uniformization property. In particular, $\Sigma_{1}^{1}$ and $\Pi_{2}^{1}$ do not have the uniformization property as we mentioned in Sect. 1.

The following results are the justification of our assumption on the uniformization property in Theorem 3.4:

Theorem 2.23 (Moschovakis [14]) Suppose that projective determinacy holds. Then for any natural number $n$ with $n \geq 1, \Pi_{2 n-1}^{1}$ and $\Sigma_{2 n}^{1}$ have the uniformization property.

Proof For the proof, see 6C (p. 310-317) in Moschovakis [15].

Theorem 2.24 (Martin and Steel [13] (Corollary, p. 91)) Suppose that there are infinitary many Woodin cardinals. Then projective determinacy holds. 


\section{Proofs of theorems}

Theorem 3.1 1. The following are equivalent:

(a) $\Sigma_{3}^{1}$-S-absoluteness holds.

(b) There is no $\boldsymbol{\Delta}_{2}^{1}$ Bernstein set.

(c) There is no $\Sigma_{2}^{1}$ Bernstein set.

(d) For any real $r$, there is a real $x$ such that $x$ is not in $\mathrm{L}[r]$.

2. Suppose that $\mathbb{P}$ is a preorder which adds a new real (i.e. there is a $\mathbb{P}$-generic filter $G$ over $V$ such that there is a real in $V[G]$ but not in $V)$. Then $\Sigma_{3}^{1}-\mathbb{P}$-absoluteness implies $\Sigma_{3}^{1}$-S-absoluteness.

Remark 3.2 We cannot replace (d) above by the statement "For any real $r$, there is a Sacks real $s$ over L $[r]$ ". For example, if we add $\omega_{1}$ many Cohen reals to $\mathrm{L}$, in that model, there is no projective Bernstein set (or even no Bernstein sets in $L(\mathbb{R})$ ) but there is no Sacks real over L.

Proof 1. By Theorem 2.8, it suffices to show that $(a) \Leftrightarrow(d)$.

First, we show that $(a) \Rightarrow(d)$.

Take any real $r$. Since Sacks forcing adds a new real (namely a Sacks real),

$$
\left.\Vdash_{\mathbb{P}} \text { “( } \exists x \in{ }^{\omega} 2\right) x \notin \mathrm{L}[\check{r}] ” .
$$

Since the reals in $\mathrm{L}[r]$ is a $\Sigma_{2}^{1}(r)$ set of reals, the above statement is equivalent to a $\Sigma_{3}^{1}$-formula with a parameter $r$. By $\Sigma_{3}^{1}$-P -absoluteness, it also holds in $V$. Hence we obtained (d).

Next, we show that $(d) \Rightarrow(a)$.

Suppose that $\Sigma_{3}^{1}$-S-absoluteness fails and we will derive a contradiction.

Then there are a Sacks real $s$ over $V$, a $\Sigma_{3}^{1}$-formula $\phi$ and a real $r$ in $V$, such that $\phi(r)$ is not absolute between $V$ and $V[s]$. By Shoenfield absoluteness (Theorem 2.17), every $\Sigma_{3}^{1}$-formula is upward absolute. Hence

$$
V[s] \vDash \phi(r) \text {, but } V \not \models \phi(r) \text {. }
$$

Let $\theta$ be the $\Sigma_{1}^{1}$-formula such that $\phi \equiv \exists^{1} \alpha_{1} \forall^{1} \alpha_{2} \theta\left(\alpha_{1}, \alpha_{2}, r\right)$. Then, there is a real $s^{\prime}$ such that for any real $y, \theta\left(s^{\prime}, y, r\right)$. Since $V \not \models \phi(r)$, by Shoenfield absoluteness, $s^{\prime}$ is not in $V$. Therefore, by Theorem 2.11 and Theorem 2.12, $V\left[s^{\prime}\right]=V[s]$ and $s^{\prime}$ is also a Sacks real over $V$. Hence in $V\left[s^{\prime}\right]$, for any real $y, \theta\left(s^{\prime}, y, r\right)$. By the forcing theorem, there is an $S$ such that

$$
S \Vdash “\left(\forall y \in{ }^{\omega} 2\right) \theta(\dot{s}, y, \check{r}) ”,
$$

where $\dot{s}$ is a canonical name for a Sacks real.

Now we go back to $V$. Let $P=\{(x, y) \mid x \in[S]$ and $\neg \theta(x, y, r)\}$. Then $\operatorname{dom}(P)=$ $[S]$ and $P$ is a $\Pi_{1}^{1}(r, S)$ set of reals. By Theorem 2.19, we can take a Shoenfield tree $T$ on $2 \times 2 \times \omega_{1}$ in $\mathrm{L}[r, S]$ such that $P=\mathrm{p}[T]$. 
The idea of proving forcing absoluteness is to approximate this $P$ by a simple set (in this case, a closed set) with a perfect set domain so that we can lift $\neg \theta$ up to a generic extension of $V$ by using Shoenfield absoluteness to contradict (*).

The following lemma, which is an analogue of 8G.1 in Moschovakis [15] due to Mansfield [12], is essential for that purpose.

Lemma 1 There is a tree $T^{\prime}$ on $2 \times 2$ and a perfect tree $S^{\prime}$ on 2 with $S^{\prime} \leq S$ such that $\left[T^{\prime}\right] \subseteq P$ and $\operatorname{dom}\left(\left[T^{\prime}\right]\right)=\left[S^{\prime}\right]$.

Proof of Lemma By exactly the same argument as 8G.1 (p. 534) in Moschovakis [15], we can construct such $T^{\prime}$ and $S^{\prime}$ unless $\mathrm{p}[T]=P$ is a subset of $\mathrm{L}[r, S]$. But this is not possible because it would imply $[S]=\operatorname{dom}(P)$ is also a subset of $\mathrm{L}[r, S]$, which would contradict (d), for every real can be coded by a real in $[S]$ and $S$.

To derive a contradiction, take a Sacks real $s^{\prime \prime}$ over $V$ with $s^{\prime \prime}$ in $\left[S^{\prime}\right]$. Since $\left[T^{\prime}\right] \subseteq$ $P=\{(x, y) \mid x \in[S]$ and $\neg \theta(x, y, r)\}$ and $\left[S^{\prime}\right]=\operatorname{dom}\left(\left[T^{\prime}\right]\right)$, the following statements hold in $V$ :

$$
\begin{aligned}
& \forall x \forall y\left((x, y) \in\left[T^{\prime}\right] \rightarrow \neg \theta(x, y, r)\right), \\
& \forall x \in\left[S^{\prime}\right] \exists y\left((x, y) \in\left[T^{\prime}\right]\right) .
\end{aligned}
$$

Since the first statement is equivalent to a $\Pi_{1}^{1}$-formula with parameters $T^{\prime}, r$ and the second statement is equivalent to a $\Pi_{2}^{1}$-formula with parameters $S^{\prime}, T^{\prime}$, by Shoenfield absoluteness, the above statements also hold in $V\left[s^{\prime \prime}\right]$. Since $s^{\prime \prime}$ is in $\left[S^{\prime}\right]$,

$$
V\left[s^{\prime \prime}\right] \vDash “\left(\exists y \in{ }^{\omega} 2\right) \neg \theta\left(s^{\prime \prime}, y, r\right) ",
$$

which contradicts $(*)$.

2. Suppose that $\mathbb{P}$ is a preorder which adds a new real. By $(d) \Rightarrow(a)$ in (1), it suffices to show (d). But we can carry out exactly the same argument as in the proof of (a) $\Rightarrow$ (d) by replacing $\mathbb{S}$ by $\mathbb{P}$.

Theorem 3.3 Let $n$ be a natural number with $n \geq 1$. If $\Sigma_{n+1}^{1}$-S-absoluteness holds, then there is no $\Delta_{n}^{1}$ Bernstein set.

Proof Take any $\Delta_{n}^{1}$ set of reals $P$. We will show that $P$ is not Bernstein, i.e. there exists a perfect tree $S$ on 2 such that either $[S] \cap P=\emptyset$ or $[S] \subseteq P$ holds.

Take a $\Sigma_{n}^{1}$-formula $\phi$, a $\Pi_{n}^{1}$-formula $\psi$, and a real $r$ such that

$$
\begin{aligned}
\forall x & \in{ }^{\omega} 2(\phi(x, r) \leftrightarrow \psi(x, r)), \\
P & =\left\{x \in{ }^{\omega} 2 \mid \phi(x, r)\right\} .
\end{aligned}
$$

Note that $(* *)$ is equivalent to a $\Pi_{n+1}^{1}$-formula with a parameter $r$. Hence, by $\Sigma_{n+1}^{1}$-S-absoluteness, $(* *)$ holds in $V[s]$ for any Sacks real $s$ over $V$.

Take any Sacks real $s$ over $V$. The following claim is essential:

Claim 1 Let $\Phi$ be a formula of the language of set theory. If $V[s] \vDash \Phi(s)$, then there is a perfect tree $S$ on 2 in $V[s]$ such that for any real $x$ in $[S] \cap V[s], V[s] \vDash \Phi(x)$. 
Proof of Claim 1 By the forcing theorem, there is an $S^{\prime}$ in $\mathbb{S} \cap V$ such that $S^{\prime} \Vdash_{\mathbb{S}} \Phi(\dot{s})$.

By Theorem 2.13, there is a perfect tree $S$ on 2 in $V[s]$ with $S \subseteq S^{\prime}$ such that for any real $s^{\prime}$ in $[S] \cap V[s], s^{\prime}$ is a Sacks real over $V$. Then by Theorem 2.11 and $S^{\prime} \Vdash \Phi(\dot{s}), V\left[s^{\prime}\right]=V[s]$ and $V\left[s^{\prime}\right] \vDash \Phi\left(s^{\prime}\right)$ for any real $s^{\prime}$ in $[S] \cap V[s]$. Hence for any real $x$ in $[S] \cap V[s], V[s] \vDash \Phi(x)$.

Suppose that $V[s] \vDash \phi(s, r)$. Then by Claim 1, there exists a perfect tree $S$ on 2 in $V[s]$, such that for any real $x$ in $[S] \cap V[s], V[s] \vDash \phi(x, r)$ On the other hand, suppose that $V[s] \vDash \neg \phi(s, r)$. Then by Claim 1, there exists a perfect tree $S$ on 2 in $V[s]$, for any real $x$ in $[S] \cap V[s], V[s] \vDash \neg \phi(x, r)$.

Hence in $V[s]$,

"There is a perfect tree $S$ on 2 such that
either $(\forall x \in[S]) \phi(x, r)$ or $(\forall x \in[S]) \neg \phi(x, r)$ holds."

By $(* *)$ in $V[s]$, the above statement is equivalent to

"There is a perfect tree $S$ on 2 such that

either $(\forall x \in[S]) \psi(x, r)$ or $(\forall x \in[S]) \neg \phi(x, r)$ holds."

This is equivalent to a $\Sigma_{n+1}^{1}$-formula with a parameter $r$. Therefore, by $\Sigma_{n+1}^{1}-\mathbb{S}$ absoluteness, the above statement also holds in $V$.

Since $P=\left\{x \in{ }^{\omega} 2 \mid \phi(x, r)\right\}$, there exists a perfect tree $S$ on 2 such that either $[S] \subseteq P$ or $[S] \cap P=\emptyset$ holds. This is what we desired.

Theorem 3.4 Let $n$ be a natural number with $n \geq 1$. Assume that $\Pi_{2 n-1}^{1}$ has the uniformization property. If there is no $\boldsymbol{\Delta}_{2 n}^{1}$ Bernstein set, then $\boldsymbol{\Sigma}_{2 n+1}^{1}-\mathbb{S}$-absoluteness holds.

Proof We will show that for any $k \leq 2 n+1, \Sigma_{k}^{1}$-S -absoluteness holds by induction on $k$. By Remark 2.18, we may assume $k \geq 3$.

Suppose that $\Sigma_{k}^{1}-\mathbb{S}$-absoluteness fails and we will derive a contradiction.

By exactly the same argument as in the proof of $(d) \Rightarrow$ (a) in Theorem 3.1 with replacing Shoenfield absoluteness by $\Sigma_{k-1}^{1}-\mathbb{S}$-absoluteness ensured by induction hypothesis, we will get a $\Sigma_{k-2}^{1}$-formula $\theta$, an $S \in \mathbb{S}$, and a real $r$ such that

$$
\begin{aligned}
& V \vDash \text { “ }(\forall x \in[S])\left(\exists y \in{ }^{\omega} 2\right) \neg \theta(x, y, r) ", \text { but } \\
& S \Vdash \text { " }\left(\forall y \in{ }^{\omega} 2\right) \theta(\dot{s}, y, \check{r}) " .
\end{aligned}
$$

Since $\{(x, y) \mid x \in[S]$ and $\neg \theta(x, y, r)\}$ is a $\Pi_{k-2}^{1}$ set, $k-2 \leq 2 n-1$, and $\Pi_{2 n-1}^{1}$ has the uniformization property, there is a $\Pi_{2 n-1}^{1}$ function $f:[S] \rightarrow{ }^{\omega} 2$ which uniformizes the set, in particular, for any real $x$ in $[S], \neg \theta(x, f(x), r)$ holds in $V$.

The idea is the same as in the proof of (d) $\Rightarrow$ (a) in Theorem 3.1. This time, we will approximate $f$ by some continuous function whose domain is a perfect subset.

The following claim provides us a local approximation of $f$ via simple sets where we use Sacks measurability instead of the non-existence of Bernstein sets. For that, we 
need that $f$ is not just a relation but a function so the uniformization property played an essential role.

Claim 2 For any $i \in \omega$, the set

$$
D_{i}=\left\{S^{\prime} \leq S \mid(\exists m \in 2)\left(\forall x \in\left[S^{\prime}\right]\right) f(x)(i)=m\right\}
$$

is dense below $S$.

Proof of Claim 2 Suppose not. Then there are a natural number $i$ and an $S^{\prime} \leq S$ such that for any $S^{\prime \prime} \leq S^{\prime}$ and for $m \in 2$, there is a real $x$ in $\left[S^{\prime \prime}\right]$ such that $f(x)(i) \neq m$. For each $m \in 2$, put $P_{m}=\{x \in[S] \mid f(x)(i)=m\}$. Then, since $f$ is a $\Pi_{2 n-1}^{1}$ subset, $P_{m}$ is a $\boldsymbol{\Delta}_{2 n}^{1}$ set of reals for each $m \in 2$. By Sacks measurability for every $\boldsymbol{\Delta}_{2 n}^{1}$ set ensured by Remark 2.6, $P_{m}$ is Sacks measurable for each $m \in 2$. Hence we can find an $S^{\prime \prime} \leq S^{\prime}$ such that $\left[S^{\prime \prime}\right] \cap P_{m}=\emptyset$ for each $m \in 2$.

Pick any element $x_{0}$ in $\left[S^{\prime \prime}\right]$. Then by the condition of $S^{\prime \prime}, x_{0}$ is not in $P_{m}$ for each $m \in 2$, namely $f\left(x_{0}\right)(i) \neq m$, which is absurd.

Now we are going to amalgamate these local approximations to produce a continuous function with a perfect set domain which is a subset of $f$. By Claim2, we can construct $\left\langle S_{t} \in \mathbb{S} \mid t \in{ }^{<\omega} 2\right\rangle$ and $\left\langle m(t) \in 2 \mid t \in{ }^{<\omega} 2\right\rangle$ such that

1. $S_{\emptyset} \subseteq S$,

2. for any $t_{1}, t_{2}$ in $<\omega 2$ with $t_{1} \subseteq t_{2}, S_{t_{1}} \supseteq S_{t_{2}}$,

3. for any $t$ in ${ }^{<\omega} 2,\left[S_{t \frown\langle 0\rangle}\right] \cap\left[S_{t \frown\langle 1\rangle}\right]=\emptyset$,

4. for any $t$ in ${ }^{<\omega} 2$ and any $x$ in $\left[S_{t}\right], f(x)(\operatorname{lh}(t))=m(t)$,

5. for any $t$ in $<\omega 2, \operatorname{lh}\left(\operatorname{stem}\left(S_{t}\right)\right) \geq \operatorname{lh}(t)$,

by induction on $\operatorname{lh}(t)$, where $\ln (t)$ is the length of $t$.

Put

$$
C=\bigcap_{i \in \omega} \bigcup_{t \in \in^{i} 2}\left[S_{t}\right] .
$$

Then, by the fusion argument, $C$ is a perfect set and $C \subseteq[S]$. Now define $g: C \rightarrow{ }^{\omega_{2}} 2$ as follows:

$$
g(x)(i)=m \text { if } m=m\left(t_{x, i}\right)
$$

where $t_{x, i}$ is the unique $t$ such that $\operatorname{lh}(t)=n$ and $x \in\left[S_{t}\right]$. It is easy to check that $g$ is continuous and a subset of $f$.

Since $g$ is continuous, $g$ is closed. Hence there are a perfect tree $S^{\prime}$ on 2 and a tree $T$ on $2 \times 2$ such that $C=\left[S^{\prime}\right]$ and $g=[T]$. The rest is exactly the same as in the proof of (d) $\Rightarrow$ (a) in Theorem 3.1 by replacing Shoenfield absoluteness by $\Sigma_{k-1}^{1}-\mathbb{S}$-absoluteness.

Remark 3.5 When $n=1$, this argument gives another proof for (b) $\Rightarrow$ (a) of 1 in Theorem 3.1 by Theorem 2.21. 
Acknowledgments The author would like to thank Joan Bagaria, through whose lecture in Kobe he became interested in forcing absoluteness. He also appreciates the help given by Yasuo Yoshinobu, who gave him useful comments on this paper and made the proof of Theorem 3.1 very simple. He is grateful to Jörg Brendle for helpful comments on Remark 3.2. Finally, he thanks the referee for several comments on this paper. This research was supported by a GLoRiClass fellowship funded by the European Commission (Early Stage Research Training Mono-Host Fellowship MEST-CT-2005-020841).

Open Access This article is distributed under the terms of the Creative Commons Attribution Noncommercial License which permits any noncommercial use, distribution, and reproduction in any medium, provided the original author(s) and source are credited.

\section{References}

1. Bagaria, J.: Definable forcing and regularity properties of projective sets of reals. Ph.D. thesis, University of California, Berkeley (1991)

2. Bartoszyński, T., Judah, H.: Set theory. On the structure of the real line. A K Peters Ltd., Wellesley (1995)

3. Brendle, J.: Amoeba-absoluteness and projective measurability. J. Symb. Log. 58(4), 1284-1290 (1993)

4. Brendle, J.: How small can the set of generics be? In: Logic Colloquium '98 (Prague), Lect. Notes Log., vol. 13, pp. 109-126. Assoc. Symbol. Logic, Urbana, IL (2000)

5. Brendle, J., Löwe, B.: Solovay-type characterizations for forcing-algebras. J. Symbol. Log. 64(3), 1307-1323 (1999)

6. Geschke, S., Quickert, S.: On sacks forcing and the sacks property. In: Classical and New Paradigms of Computation and their Complexity Hierarchies. Trends Log. Stud. Log. Libr., vol. 23, pp. 95-139. Kluwer Acad. Publ., Dordrecht (2004)

7. Halbeisen, L., Judah, H.: Mathias absoluteness and the Ramsey property. J. Symbol. Log. 61(1), 177194 (1996)

8. Jech, T.: Set theory. Springer Monographs in Mathematics. Springer-Verlag, Berlin (2003). The third millennium edition, revised and expanded

9. Judah, H.: Absoluteness for projective sets. In: Logic Colloquium '90 (Helsinki, 1990). Lecture Notes Logic, vol. 2, pp. 145-154. Springer, Berlin (1993)

10. Judah, H., Shelah, S.: $\Delta_{2}^{1}$-sets of reals. Ann. Pure Appl. Log. 42(3), 207-223 (1989)

11. Kondô, M.: Sur l'uniformisation des complémentaires analytiques et les ensembles projectifs de la seconde classe. Japan J. Math. 15, 197-230 (1939)

12. Mansfield, R.: Perfect subsets of definable sets of real numbers. Pacific J. Math. 35, $451-457$ (1970)

13. Martin, D.A., Steel, J.R.: A proof of projective determinacy. J. Am. Math. Soc. 2(1), $71-125$ (1989)

14. Moschovakis, Y.N.: Uniformization in a playful universe. Bull. Am. Math. Soc. 77, 731-736 (1971)

15. Moschovakis, Y.N.: Descriptive Set Theory. Studies in Logic and the Foundations of Mathematics. Vol. 100, North-Holland Publishing Co, Amsterdam (1980)

16. Sacks, G.E.: Forcing with perfect closed sets. In: Axiomatic Set Theory (Proc. Sympos. Pure Math., vol. XIII, Part I, Univ. California, Los Angeles, Calif., 1967), pp. 331-355. Amer. Math. Soc., Providence, R.I. (1971)

17. Shelah, S.: Can you take Solovay's inaccessible away? Israel J. Math. 48(1), 1-47 (1984)

18. Shoenfield, J.R.: The problem of predicativity. In: Essays on the Foundations of Mathematics, pp. 132139. Magnes Press, Hebrew Univ., Jerusalem (1961)

19. Solovay, R.M.: On the cardinality of $\Sigma_{2}^{1}$ sets of reals. In: Foundations of Mathematics (Symposium Commemorating Kurt Gödel, Columbus, Ohio, 1966), pp. 58-73. Springer, New York (1969)

20. Woodin, W.H.: On the consistency strength of projective uniformization. In: Proceedings of the Herbrand symposium (Marseilles, 1981). Stud. Logic Found. Math., vol. 107, pp. 365-384. North-Holland, Amsterdam (1982) 